\title{
Does Emotion Influence the Use of Auto-suggest during Smartphone Typing?
}

\author{
Surjya Ghosh \\ IIT Kharagpur, India \\ surjya.ghosh@iitkgp.ac.in
}

\author{
Kaustubh Hiware \\ IIT Kharagpur, India \\ hiwarekaustubh@iitkgp.ac.in
}

\author{
Niloy Ganguly \\ IIT Kharagpur, India \\ niloy@cse.iitkgp.ernet.in
}

\author{
Bivas Mitra \\ IIT Kharagpur, India \\ bivas@cse.iitkgp.ernet.in
}

\author{
Pradipta De \\ Georgia Southern University, USA \\ pde@georgiasouthern.edu
}

\begin{abstract}
Typing based interfaces are common across many mobile applications, especially messaging apps. To reduce the difficulty of typing using keyboard applications on smartphones, smartwatches with restricted space, several techniques, such as auto-complete, autosuggest, are implemented. Although helpful, these techniques do add more cognitive load on the user. Hence beyond the importance to improve the word recommendations, it is useful to understand the pattern of use of auto-suggestions during typing. Among several factors that may influence use of auto-suggest, the role of emotion has been mostly overlooked, often due to the difficulty of unobtrusively inferring emotion. With advances in affective computing, and ability to infer user's emotional states accurately, it is imperative to investigate how auto-suggest can be guided by emotion aware decisions. In this work, we investigate correlations between user emotion and usage of auto-suggest i.e. whether users prefer to use auto-suggest in specific emotion states. We developed an Android keyboard application, which records auto-suggest usage and collects emotion self-reports from users in a 3-week in-the-wild study. Analysis of the dataset reveals relationship between user reported emotion state and use of auto-suggest. We used the data to train personalized models for predicting use of auto-suggest in specific emotion state. The model can predict use of auto-suggest with an average accuracy (AUCROC) of $82 \%$ showing the feasibility of emotion-aware auto-suggestion.
\end{abstract}

\section{CCS CONCEPTS}

- Human-centered computing $\rightarrow$ Keyboards; Smartphones; Human computer interaction (HCI); User interface design.

\section{KEYWORDS}

Auto-suggestion; Emotion; Keyboard; Smartphone

Permission to make digital or hard copies of all or part of this work for personal or classroom use is granted without fee provided that copies are not made or distributed for profit or commercial advantage and that copies bear this notice and the full citation on the first page. Copyrights for components of this work owned by others than ACM must be honored. Abstracting with credit is permitted. To copy otherwise, or republish, to post on servers or to redistribute to lists, requires prior specific permission and/or a fee. Request permissions from permissions@acm.org.

IUI '19, March 17-20, 2019, Marina del Rey, CA, USA

(c) 2019 Association for Computing Machinery.

ACM ISBN 978-1-4503-6272-6/19/03 . \$ \$15.00

https://doi.org/10.1145/3301275.3302329
ACM Reference Format:

Surjya Ghosh, Kaustubh Hiware, Niloy Ganguly, Bivas Mitra, and Pradipta De. 2019. Does Emotion Influence the Use of Auto-suggest during Smartphone Typing?. In 24th International Conference on Intelligent User Interfaces (IUI '19), March 17-20, 2019, Marina del Rey, CA, USA. ACM, New York, NY, USA, 6 pages. https://doi.org/10.1145/3301275.3302329

\section{INTRODUCTION}

Keyboard applications on small devices, like smartphones, smartwatches, are still an essential interface for many applications, such as WhatsApp, Facebook Messenger, Google Hangout [22]. However, typing on small devices come with their challenges due to limited space. Several techniques like auto-suggest, auto-complete are designed to reduce the amount of typing, but require additional space to display the word suggestions. It also requires the user to read, parse, and choose from the suggestions, adding to the cognitive load, and disrupting the flow of typing [20]. Can auto-suggestions be adaptive, and displayed only when the user is most likely to use them? By making the auto-suggestions adaptive, it is possible to optimize the keyboard layout design and improve the user experience during text input interaction.

In literature, different techniques like alternative layouts $[4,9$, 27], gesture keyboards [1, 17, 21], key-target resizing [14], sensorbased adaptation [3, 13] and phrase recommendations [2] have been adopted to improve typing performance in these small touch based devices. The influence of mental state on typing speed and accuracy in smartphone has also been established $[10,11]$. However, the role of emotion has largely been overlooked in deciding autosuggest usage frequency. We note that the performance of many recommender systems like automated tutoring systems [19], movie recommendation [5], music recommendation [8] can benefit by considering user's emotion, leading to affect-aware designs. Moreover, many affect determination techniques have been designed recently which can determine user emotion unobtrusively $[7,12,16,18,26]$. These advances in affective computing and the presence of affective recommender systems in other areas motivate us to take a deeper look at the role of user emotion on auto-suggest usage to improve overall typing performance.

We, in this paper, investigate if there is any relationship between emotion state and auto-suggest usage i.e. whether users prefer to use auto-suggest in a specific emotion state. We develop an Android based keyboard application, which traces user's touch interactions (typing, swyping) during text entry and automatically 
suggest words based on the entered initial characters. The application also collects self-reported emotion labels associated with the text entry sessions. We distinguish between these sessions, where the user has accepted or skipped auto-suggestions and find the correlation between emotion states and auto-suggest usage. We observe a strong relation between these two indicating emotion indeed plays a role to decide whether a user is going to accept or skip the auto-suggested words. This led to the development of a personalized machine learning model, which detects whether a user is going to use auto-suggest during a typing session.

We carry out a 3-week study involving 13 participants and ask them to use the keyboard application for typing activities and recording emotion states. We have collected approximately 3000 sessions, where the users have either used or skipped the autosuggested words. Our key results demonstrate that based on user emotion it is possible to determine auto-suggest usage with an average accuracy (AUCROC) of $82 \%$ (std dev. 9\%). It indicates the possibility to consider human emotion also while devising autosuggestion algorithms to improve typing performance.

\section{METHODOLOGY}

\subsection{Apparatus Design}

We need to log when a user accepts auto-suggestion, what is her perceived emotion. Since it is infeasible to prompt a user to record an emotion after every use of auto-suggest, we design it based on typing sessions. A typing session is an uninterrupted typing activity within one application. As shown in Figure 1, time period $t 1-t 2$, and $t 3-t 4$, denote two sessions. While the user types each character, we use an English dictionary to suggest words. The user selects a word from the list, or ignores the suggestion. At the end of text entry in a session, when user changes the application, she is immediately prompted to record her perceived emotion during the session. The probing is done as soon as the application is changed so that perceived emotion is less likely to alter. The user provided emotion self-report is associated with this text entry session. We design an Android based keyboard application that implements the two necessary features, viz. (a) displaying word suggestions based on typing and labeling auto-suggestion usage (b) collecting emotion self-reports.

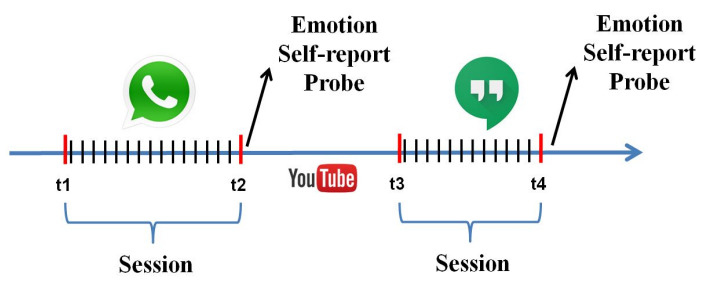

Figure 1: Schematic of auto-suggestion scenario. In a session (e.g. time interval between $t 1$ and $t 2$ ) user performs key pressing events (denoted by small bar) and accordingly suggestions are shown. At the end of text entry in the session, emotion self-report is collected and attached to the session.

\subsection{Experiment Apparatus}

We have designed the keyboard app based on Android Input Method Editor (IME) facility. It is same as QWERTY keyboard with additional capability of tracing user's touch interaction activities (typing, swyping) during text entry. We do not store any alphanumeric character because of privacy reason and collect only the timestamp of each touch event. We show the keyboard interface in Figure 2.

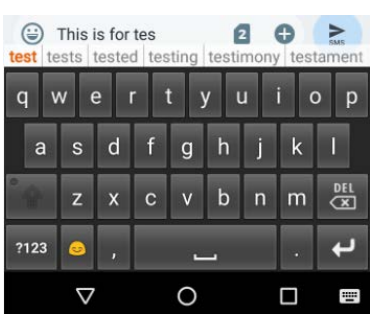

Figure 2: App keyboard

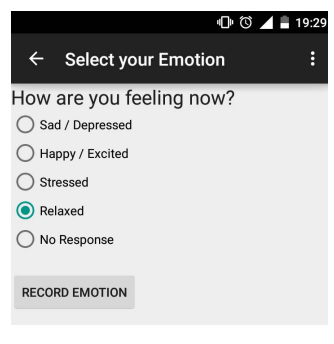

Figure 3: Emotion collection UI
Labeling Auto-suggestion Usage: Once a user accepts a word from the list of suggested words, we store the timestamp of the auto-suggest usage. If the user accepts at least one auto-suggestion in a session, the session is labeled as accepted and if she ignores all suggestions in a session, it is marked as skipped. In our view, even a single use of auto-suggest in a session implies that user finds it useful, and auto-suggestion should be active for such sessions.

Collecting Emotion Self-report: Additionally, we also collect self-reported emotion labels from users. Once user completes typing from an application and switches the application, we probe her to report the felt emotion during the session. We ask her to report one of the following four emotion states (happy, sad, stressed, relaxed) as shown in Figure 3. We select these emotion states based on the Circumplex model of emotion [23], as they represent largely represented emotion from separate quadrants, which makes selfreporting easier for the user. We keep the interface simple by explicitly recording emotion and do not consider the intensity of perceived emotion, which can make self-reporting difficult. We also keep the provision of No Response so that user can skip emotion recording by selecting this.

\subsection{Field Study}

We have recruited 20 university students ( 15 male, 5 female) aged between 20 to 35 years. We have installed the application on their smartphones and asked them to use it for 3 weeks for typing activity and emotion self-reporting. We have also showed them how to select a word from list of auto-suggested words that appear during typing. It was also instructed that once they complete typing and change the application, they may receive an emotion self-report collection UI, where they have to record their perceived emotion during text recording. Finally, we have selected 13 users (10 male, 3 female), who have accepted auto-suggestions in at least 20 sessions.

\section{DATASET}

We have collected a total of 3284 typing sessions from the field study, out of which 330 sessions $(\approx 10 \%)$ are tagged as No Response. 
We have eliminated these sessions and used remaining 2954 typing sessions for evaluation. On average, we obtain 227 sessions (std. $\operatorname{dev} 151.7)$ per user. Out of these, there are 841 sessions, which are labeled as accepted and rest are labeled as skipped. We show the fraction of accepted and skipped sessions for every user in Figure 4. It is observed that majority of the users have less number of accepted sessions than skipped sessions. On average in $28 \%$ sessions, users have accepted auto-suggestions.

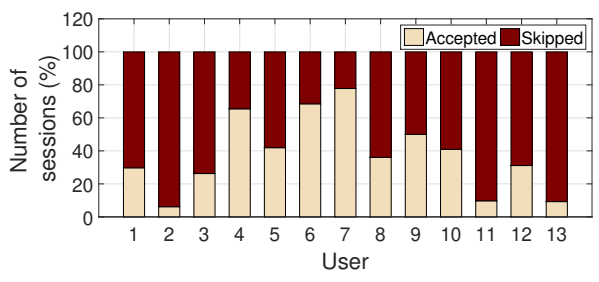

Figure 4: Frequency distribution of accepted and skipped sessions corresponding to every user. Most of the users have less accepted sessions resulting in overall $28 \%$ accepted sessions.

\subsection{Auto-suggest Usage based on Emotion}

We investigate the correlation between perceived emotion and autosuggest adoption in this section. First, we compare the usage of auto-suggest corresponding to every emotion label in Figure 5. We observe that at every emotion state the usage of auto-suggest is low, however users are more likely to accept auto-suggest in $\mathrm{sad}$, relaxed state. However, we also observe that in these two states there is a high amount of auto-suggest rejections, which may be due to other factors like - users did not find suitable word due to limited dictionary size.

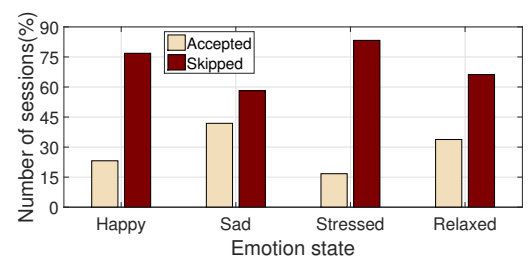

Figure 5: Frequency distribution of accepted and skipped sessions corresponding to every emotion. Users are more likely to use auto-suggest in sad, relaxed state.

Next, we investigate whether this observation holds true for every user. For this purpose, we compare the frequency distribution of emotion states for accepted and skipped sessions of each user. We compute the fraction of each emotion label (happy, sad, stressed, relaxed) for accepted and skipped session of every user and plot the same in Figure 6.

We observe that majority of the users prefer to use auto-suggest in relaxed state. We also observe users like $U 2, U 7$ accept autosuggestions heavily in sad state. At the same time few users prefer suggestions in happy, stressed state. While these observations largely corroborate with earlier finding, we investigate further if auto-suggest usage preference differs for individual user. In Figure

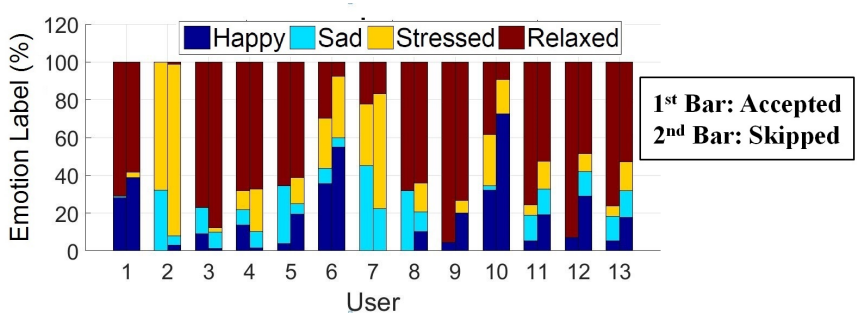

Figure 6: Comparison of frequency distribution of emotion states for accepted and skipped sessions. First bar shows the distribution of emotions for accepted sessions, while the second one shows the same for skipped sessions.

6 , we observe that users like $U 6, U 10$, are having observable difference in frequency distribution of accepted and skipped sessions, but we validate the same with statistical test. For this purpose, we investigate if there is any significant difference in frequency distribution of emotion state for accepted and skipped sessions using Chi-square test [24].

\begin{tabular}{|l|c|c|c|c|c|c|c|}
\hline & U1 & U2 & U3 & U4 & U5 & U6 & U7 \\
\hline \hline df & 3 & 3 & 3 & 3 & 3 & 3 & 3 \\
\hline Chi-square stat & 11.665 & 32.213 & 14.095 & 9.769 & 12.424 & 9.19 & 9.994 \\
\hline p-value & 0.0086 & 0.0000 & 0.0028 & 0.0206 & 0.0061 & 0.0269 & 0.0186 \\
\hline & U8 & U9 & U10 & U11 & U12 & U13 & - \\
\hline \hline df & 3 & 3 & 3 & 3 & 3 & 3 & - \\
\hline Chi-square stat & 9.25 & 8.74 & 37.571 & 9.001 & 16.559 & 9.015 & - \\
\hline p-value & 0.0262 & 0.0329 & 0.0000 & 0.0293 & 0.0009 & 0.0291 & - \\
\hline
\end{tabular}

Table 1: For every user, the frequency distribution of emotions for accepted and skipped sessions is found to be significantly $(p<0.05)$ different using Chi square test.

The null hypothesis is that there is no significant difference in frequency distribution of emotion states for accepted and skipped sessions. To test the hypothesis, we count the number of accepted sessions tagged with different emotions and the same for skipped sessions. Then we perform the Chi-square test to find if there is any significant difference in frequency distribution for accepted and skipped sessions. We observe the frequency distribution of emotion states associated with accepted and skipped session varies significantly $(p<0.05)$ for every user (Table 1$)$. This indicates that there is difference in the frequencies of perceived emotion when a user accepts or skips auto-suggestions. This finding and the observations from Figure 6 reinforce that individual preference for auto-suggest usage differ based on emotion, which drive us to design a personalized auto-suggest usage prediction model.

\section{MODEL CONSTRUCTION}

The auto-suggest usage prediction model determines whether the user will accept the suggestions or not in a session, thus it becomes a binary class prediction problem. In the collected dataset, sessions are labeled as accepted, skipped; from which we extract the emotion related features.

We use the features as defined in Table 2 to build the model. Emotion $_{\text {curr }}$ refers to the associated emotion label with current session while Emotion prev refers to the emotion recorded by the user in the immediate previous session. We decide to use the emotion associated with immediate previous session only, as the effect 


\begin{tabular}{|l|l|}
\hline Feature name & Feature description \\
\hline Emotion $_{\text {curr }}$ & Emotion associated with current session \\
\hline Emotion $_{\text {prev }}$ & Emotion associated with previous session \\
\hline Time $_{\text {elapsed }}$ & $\begin{array}{l}\text { Elapsed time between previous and current } \\
\text { session emotion recording timestamp }\end{array}$ \\
\hline
\end{tabular}

Table 2: Features used for auto-suggest usage prediction

of emotion persists over time and it fades way with time [11, 25] resulting the immediate previous emotion having the highest impact among all previously recorded emotions. However, we capture the fading effect of emotion using Time elapsed $_{\text {feature, which mea- }}$ sures the elapsed time between the emotion recording timestamp of previous session and the current session. We build a personalized machine learning model for every user. We create the models using the Random Forest supervised machine learning algorithm as implemented in Weka [15].

\section{EVALUATION}

We evaluate the model using 10 -fold cross validation and measure the model performance using AUCROC (Area under the Receiver Operating Characteristic curve) and F-score. We also compare the performance of the proposed model with following baseline autosuggest usage prediction models.

(i) Most Represented Emotion (MRE): We observe that for most of the users there is one emotion, in which she accepts most of the autosuggestions (Figure 6). As a result, we develop this personalized model, which recommends suggestions whenever it detects this emotion state and otherwise not.

(ii) Generalized (GEN): We develop this model aggregating all users' data and use the same set of features. We use leave-one-participantout-cross-validation to test this model i.e. we build the model for one user using data from other users and test the model using this user's data. If this model is effective in determining the auto-suggest usage, then it can reduce the overhead of personalized training.

\subsection{Auto-suggest Usage Prediction}

We show the auto-suggest usage prediction result in Figure 7 for every user. We obtain an average AUCROC of $73 \%$ (std. dev 9\%) and F-score of $77 \%$ (std. dev 7\%).

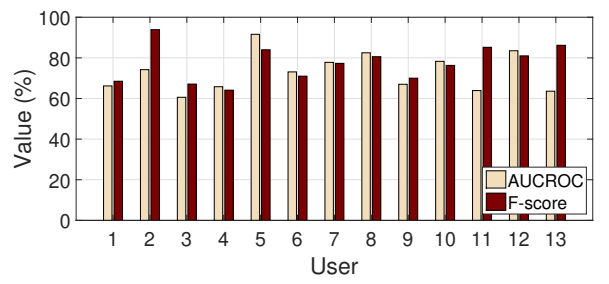

Figure 7: Auto-suggest usage prediction model performance.

We observe that for 7 out of 13 users, AUCROC is greater than or equal to $70 \%$ and for 12 out of 13 users the value of AUCROC is greater than or equal to $65 \%$. It is also noted that for 10 out of 13 users, F-score is greater than $70 \%$. These findings reveal that the model can detect auto-suggest usage accurately for the users.

\subsection{Comparison with Baselines}

In Figure 8, we compare the performance of the proposed personalized model with other two baseline models. We observe that it outperforms both of them. The personalized model based on most represented emotion only (MRE) attains significantly poor average AUCROC value of $52 \%$ (standard deviation $7 \%$ ). However, the generalized model (GEN) attains comparatively better performance (average AUCROC of 59\%) with high standard deviation of $19 \%$.

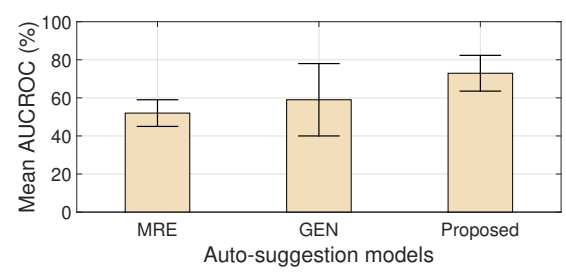

Figure 8: Mean AUCROC for different models. Error bar indicates std dev. Proposed model outperforms the baseline models.

These observations indicate that always predicting the usage of auto-suggest based on most represented emotion is not a good choice. Similarly, aggregating all user's emotion data to build the model is not a good design choice either. We observe a high standard deviation (19\%) in user-wise AUCROC for this model. This indicates that different users prefer to use auto-suggest at different emotion (reinforcing the finding of Figure 6), as a result the general model does not perform well. In summary, considering the individual emotion while devising the auto-suggest prediction model is the best option.

\subsection{Feature Importance}

We measure the effectiveness of different features using information gain (IG). We use the InfoGainAttributeEval method from WEKA [15] to derive the information gain (IG) of each attribute. Table 3 shows the average ranking of the features. The feature evaluation used 10 -fold cross validation.

\begin{tabular}{|l|c|c|}
\hline Feature & Rank & Avg. IG \\
\hline \hline Emotion $_{\text {curr }}$ & 1 & 0.1194 \\
\hline Emotion $_{\text {prev }}$ & 2 & 0.1098 \\
\hline Time $_{\text {elapsed }}$ & 3 & 0.0794 \\
\hline
\end{tabular}

Table 3: Discriminating features based on Information Gain. Emotion associated with current session (Emotion $_{\text {curr }}$ ) is found to be the most significant.

We observe that Emotion curr $_{\text {is }}$ the most discriminating factor

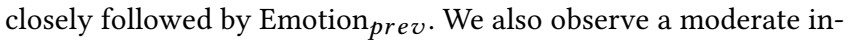
fluence of Time elapsed $_{\text {. This is intuitive as the emotion state often }}$ persists over time and the effect of previous emotion fades away with higher elapsed time.

\subsection{Countering Class Imbalance}

We observe in Figure 4 that the distribution of accepted and skipped classes are skewed, which impacts the overall classification performance. So, we overcome the problem of class imbalance using 
Synthetic Minority Over-sampling Technique (SMOTE) [6]. Using SMOTE, we oversample the class with fewer number records so that both the classes contain almost equal number of records.

We compare the difference in classification performance for the two cases - unbalanced dataset and balanced dataset. The average accuracy (AUCROC) is $73 \%$ for the original unbalanced dataset, while it is $82 \%$ after balancing the data using SMOTE. We also report the F-score for each class (accepted and skipped) of both datasets in Figure 9.

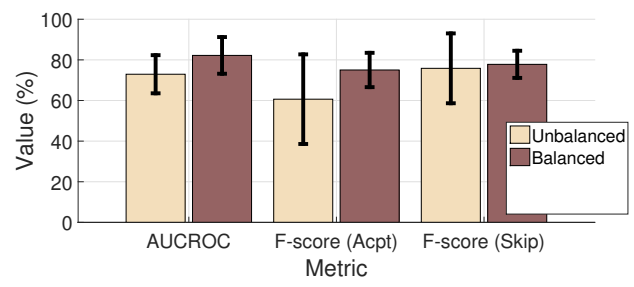

Figure 9: Auto-suggest prediction performance comparison between unbalanced and balanced data. The performance improves with balanced dataset.

We observe that both overall and class-wise performance improves after balancing the dataset. It also shows that the standard deviation reduces (AUCROC by $1 \%$, F-score for accepted class by $14 \%$, F-score for skipped class by $10 \%$ ) after balancing the dataset i.e. variation in user-wise performance also reduces. This shows that the proposed model can attain high classification performance with adequate data.

\section{DISCUSSION}

Our results show that based on emotion, it is possible to determine whether the user to going to use auto-suggest in a session. This opens the scope to develop affect-aware smartphone keyboard, which can optimize the keyboard layout by dynamically displaying or hiding the space reserved for auto-suggestion based on user emotion.

In this paper, we investigate the relationship between autosuggest usage and user emotion only. So, we consider only emotion related features to build the model. However, other features can also be explored to develop the auto-suggest usage prediction model.

With regard to increase the precision of auto-suggestions, there may be different approaches e.g. using larger dictionary, using the application or task type information. However, in this work we have not considered to improve the quality of auto-suggestions and left the same as future work.

\section{CONCLUSION}

In this paper, we investigate if user perceived emotion influences the use of auto-suggest, which aims at improving typing performance in small touch based devices. We develop an Android based keyboard application, which provides suggestions to users during typing and collects four types of emotion self-reports (happy, sad, stressed, relaxed) in parallel. We collect auto-suggest usage and emotion self-report details using this app from a 3-week study. Our analysis on this dataset reveals a strong relation between user emotion and auto-suggest usage indicating users are more likely to use autosuggest in sad or relaxed state. Driven by this finding, we develop a machine learning model, which predicts auto-suggest usage based on emotion with an average accuracy of $82 \%$.

\section{REFERENCES}

[1] Ouais Alsharif, Tom Ouyang, Françoise Beaufays, Shumin Zhai, Thomas Breuel, and Johan Schalkwyk. 2015. Long short term memory neural network for keyboard gesture decoding. In 2015 IEEE International Conference on Acoustics, Speech and Signal Processing (ICASSP). IEEE, 2076-2080.

[2] Kenneth C Arnold, Krzysztof Z Gajos, and Adam T Kalai. 2016. On Suggesting Phrases vs. Predicting Words for Mobile Text Composition. In Proceedings of the 29th Annual Symposium on User Interface Software and Technology. ACM, 603-608.

[3] Shiri Azenkot and Shumin Zhai. 2012. Touch behavior with different postures on soft smartphone keyboards. In Proceedings of the 14th international conference on Human-computer interaction with mobile devices and services. ACM, 251-260.

[4] Xiaojun Bi, Barton A Smith, and Shumin Zhai. 2010. Quasi-qwerty soft keyboard optimization. In Proceedings of the SIGCHI Conference on Human Factors in Computing Systems. ACM, 283-286.

[5] Luca Canini, Sergio Benini, and Riccardo Leonardi. 2013. Affective recommendation of movies based on selected connotative features. IEEE Transactions on Circuits and Systems for Video Technology 23, 4 (2013), 636-647.

[6] Nitesh V. Chawla, Kevin W. Bowyer, Lawrence O. Hall, and W. Philip Kegelmeyer. 2002. SMOTE: synthetic minority over-sampling technique. Fournal of artificial intelligence research 16 (2002), 321-357.

[7] M. Ciman and K. Wac. 2018. Individuals' stress assessment using humansmartphone interaction analysis. IEEE Transactions on Affective Computing 9, 1 (2018), 51-65.

[8] James J Deng, Clement HC Leung, Alfredo Milani, and Li Chen. 2015. Emotional states associated with music: Classification, prediction of changes, and consideration in recommendation. ACM Transactions on Interactive Intelligent Systems (TiiS) 5, 1 (2015), 4.

[9] Mark Dunlop and John Levine. 2012. Multidimensional pareto optimization of touchscreen keyboards for speed, familiarity and improved spell checking. In Proceedings of the SIGCHI Conference on Human Factors in Computing Systems. ACM, 2669-2678.

[10] Surjya Ghosh, Niloy Ganguly, Bivas Mitra, and Pradipta De. 2017. Evaluating Effectiveness of Smartphone Typing as an Indicator of User Emotion. In 2017 Seventh International Conference on Affective Computing and Intelligent Interaction (ACII). IEEE, 146-151.

[11] Surjya Ghosh, Niloy Ganguly, Bivas Mitra, and Pradipta De. 2017. TapSense: Combining Self-Report Patterns and Typing Characteristics for Smartphone based Emotion Detection. In Proceedings of the 19th International Conference on Human-Computer Interaction with Mobile Devices and Services (MobileHCI). ACM, 2.

[12] Surjya Ghosh, Niloy Ganguly, Bivas Mitra, and Pradipta De. 2018. Poster: Effectiveness of Deep Neural Network Model in Typing-based Emotion Detection on Smartphones. In Proceedings of the 24th Annual International Conference on Mobile Computing and Networking. ACM, 750-752.

[13] Mayank Goel, Alex Jansen, Travis Mandel, Shwetak N Patel, and Jacob O Wobbrock. 2013. ContextType: using hand posture information to improve mobile touch screen text entry. In Proceedings of the SIGCHI conference on human factors in computing systems. ACM, 2795-2798.

[14] Asela Gunawardana, Tim Paek, and Christopher Meek. 2010. Usability guided key-target resizing for soft keyboards. In Proceedings of the 15th international conference on Intelligent user interfaces. ACM, 111-118.

[15] Mark Hall, Eibe Frank, Geoffrey Holmes, Bernhard Pfahringer, Peter Reutemann, and Ian H. Witten. 2009. The WEKA Data Mining Software: An Update. SIGKDD Explor. Newsl. 11, 1 (Nov. 2009), 10-18.

[16] Robert LiKamWa, Yunxin Liu, Nicholas D Lane, and Lin Zhong. 2013. Moodscope: Building a mood sensor from smartphone usage patterns. In Proceeding of the 11 th annual international conference on Mobile systems, applications, and services (Mobisys). ACM, 389-402.

[17] Anders Markussen, Mikkel Rønne Jakobsen, and Kasper Hornbæk. 2014. Vulture: a mid-air word-gesture keyboard. In Proceedings of the 32nd annual ACM conference on Human factors in computing systems. ACM, 1073-1082.

[18] Martin Pielot, Tilman Dingler, Jose San Pedro, and Nuria Oliver. 2015. When attention is not scarce-detecting boredom from mobile phone usage. In Proceedings of the 2015 ACM international joint conference on pervasive and ubiquitous computing. ACM, 825-836.

[19] Kaśka Porayska-Pomsta, Manolis Mavrikis, and Helen Pain. 2008. Diagnosing and acting on student affect: the tutor's perspective. User Modeling and User-Adapted Interaction 18, 1-2 (2008), 125-173.

[20] Philip Quinn and Shumin Zhai. 2016. A cost-benefit study of text entry suggestion interaction. In Proceedings of the 2016 CHI conference on human factors in computing systems. ACM, 83-88. 
[21] Shyam Reyal, Shumin Zhai, and Per Ola Kristensson. 2015. Performance and user experience of touchscreen and gesture keyboards in a lab setting and in the wild. In Proceedings of the 33rd Annual ACM Conference on Human Factors in Computing Systems. ACM, 679-688.

[22] Sherry Ruan, Jacob O Wobbrock, Kenny Liou, Andrew Ng, and James A Landay. 2018. Comparing Speech and Keyboard Text Entry for Short Messages in Two Languages on Touchscreen Phones. Proceedings of the ACM on Interactive, Mobile, Wearable and Ubiquitous Technologies 1, 4 (2018), 159

[23] James A Russell. 1980. A circumplex model of affect. fournal of Personality and Social Psychology 39, 6 (1980), 1161-1178.

[24] Ronald J Tallarida and Rodney B Murray. 1987. Chi-square test. In Manual of Pharmacologic Calculations. Springer, 140-142.
[25] Philippe Verduyn and Saskia Lavrijsen. 2015. Which emotions last longest and why: The role of event importance and rumination. Motivation and Emotion 39, 1 (2015), 119-127.

[26] Katarzyna Wac, Matteo Ciman, and Ombretta Gaggi. 2015. iSenseStress: Assessing Stress Through Human-Smartphone Interaction Analysis. In Proceedings of the 9th International Conference on Pervasive Computing Technologies for Healthcare. ICST (Institute for Computer Sciences, Social-Informatics and Telecommunications Engineering), 84-91.

[27] Shumin Zhai and Per Ola Kristensson. 2008. Interlaced QWERTY: accommodating ease of visual search and input flexibility in shape writing. In Proceedings of the SIGCHI Conference on Human Factors in Computing Systems. ACM, 593-596. 